\title{
Gas Distribution System for Optimization of the Technological Process of Coating in an Unbalanced Magnetron Sputtering Installation
}

\author{
Ivan Uzunov \\ Central Laboratory of Applied Physics, Bulgarian Academy \\ of Sciences \\ Plovdiv, Bulgaria \\ vanlu@mail.bg
}

\author{
Angel Lengerov \\ Technical University - Sofia, Plovdiv Branch \\ Plovdiv, Bulgaria \\ anlen@tu-plovdiv.bg
}

\begin{abstract}
The paper proposes a new design solution for regulating the distribution of the working gases in the vacuum chamber of the installation (UDP -850/4) for unbalanced magnetron sputtering, which will improve the mode and quality of application of multilayer nano-coatings. Constructive documentation for manufacturing a gas distribution system has been prepared, as well as instructions for assembly to the vacuum chamber.
\end{abstract}

Keywords optimization, distribution, gas, vacuum chamber, magnetron sputtering

\section{INTRODUCTION}

The technological coating process in an unbalanced magnetron sputtering installation (UDP - 850/4) is a wellknown modern method for depositing thin layers on substrates (details) in order to improve their performance $[8,9,10]$. This is a vacuum technology (nanotechnology), based mainly on physical processes. The processes take place in a vacuum environment, under conditions of artificially and purposefully created argon plasma.

Vacuum deposition methods, whether physical or chemical, have some significant advantages, such as high level of purity, very good adhesion to the substrate, good uniformity and density of the layers, small residual stresses and microdefects in the structure, possibility for deposition of ultra-thin layers, very good possibilities for controlling the thickness, structure, mechanical stresses and other parameters of the layers, all of these leading to high quality of the obtained coatings $[3,9,10]$.

The ion-plasma method includes diode and magnetron sputtering. The principle of operation in magnetron sputtering is shown in fig. $1[5,6]$.
In magnetron sputtering, ionized argon fires at the target, releasing atoms, which, in turn, form layers on the substrate. The electrons and the argon ions form a plasma close to the target, due to the magnetic field. As a result of the magnetic action, the electrons move in a spiral along the magnetic field lines.

An important advantage of magnetron sputtering systems is the high speed of coating application, as well as the possibility for obtaining thin layers of uniform thickness on substrates with a considerable area.

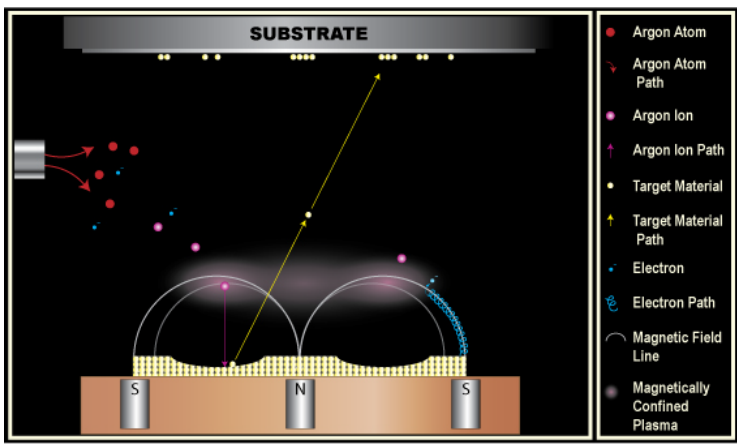

Fig.1. Magnetron sputtering [6]

Studies show that the main interrelated parameters, determining the rate of sputtering of the targets, are the discharge voltage, the discharge current, the induction of the magnetic field near the cathode surface, and the working gas pressure $[5,6]$. In these systems the inert gas argon is usually used in the role of a working gas. 
Both the working gas argon (Ar) and the active gas are supplied to the vacuum space by pipelines. It is known that the size of the inlet and outlet openings of the pipeline, as well as the passability (the design features) of the individual elements of the vacuum gas system have a significant influence on the movement of the gas $[1,2,4$, 7].

In the considered vacuum chamber - part of the installation UDP - 850/4 - a vertical gas pipe with 4 or 6 openings is used, through which the working gases $A r$ and, in this case $N_{2}$, pass, to be used in processes of applying coatings by means of unbalanced magnetron sputtering. Fig. 2.

The pipe is located between the third (on the right side of the heater) and the fourth target. This is a T-shaped tubular structure (pipe 6,3x4), in which the short, transversely welded pipe, serves for connection (inlet) to the gas supply system of the installation and, through the openings of the vertical pipe (ø 1), blocked at both its ends by a stopper - bolt - DIN 912 M5 x $8 \ldots 8$ N, the gases enter the working space.

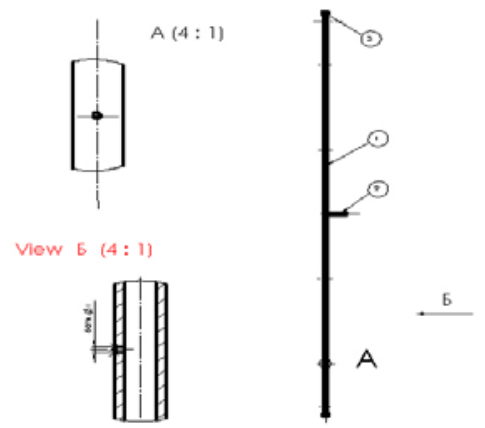

Fig. 2. Operating gas distribution system (gas line)

1 - vertical gas distribution pipe; 2 - transverse connection pipe; 3 - stopper - 2 pcs - bolt - DIN 912 M5 x 8

\section{MEthodOLOGY}

The chamber uses different types of targets - Ti, Cr and Al. Each material has a different sputtering rate and forms stoichiometric nitride compounds using different amounts of nitrogen. Accordingly, the moment of occurrence of poisoning (deposition of harmful - nitride layers on the target material) of the targets is related to different amounts of nitrogen. On the other hand, in a vacuum environment, the movement of gases is inert and their distribution is nonuniform. Therefore, it is necessary to build an individual line for supplying each target in the chamber with working gases, with an option to change the number of openings depending on whether the given material needs less or more nitrogen.

The present work aims at designing and preparation of constructive documentation for building a new gas line, consisting of several parts and allowing the working gases used in the vacuum chamber (Fig. 3) to reach each of the targets - the $2^{\text {nd }}, 3^{\text {rd }}$ and $4^{\text {th }}$ - in regulated amounts.

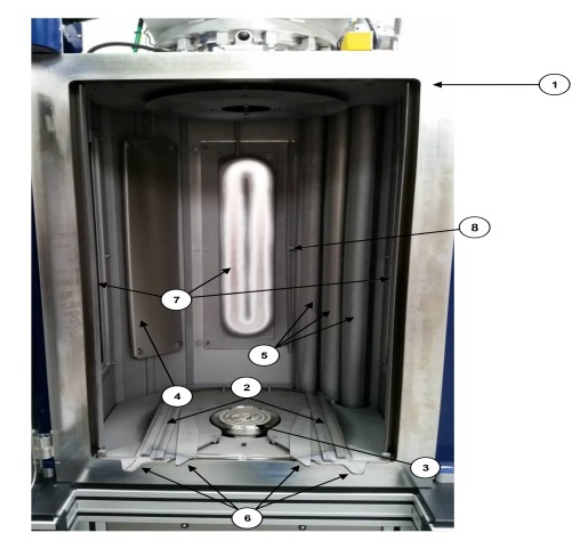

Fig.3. Vacuum chamber of a vacuum coating installation - (UDP 850/4): 1 - Vacuum chamber; 2 - guides when moving the carousel; 3 main carousel drive; 4 - heater (flat, infrared);

5 - thermocouples; 6 - locking lever system; 7 - 2nd, 3rd and 4th targets (from left to right); 8 - operating gas line

The realization of this aim is achieved by solving the following tasks:

1. The first section of the gas line must measure up to the current gas pipe on which stoppers can be placed.

2. A second section must be added to this main section. The second section must be a continuation of the first one and go in the base of the chamber, outside the carousel and under the heater.

3. The gas distribution system should consist of two separate outlet openings with stoppers, acting as additional connections for target 2 and target 4, respectively.

\subsection{Design brief} are set:

The following main design parameters of the system

- the gas pipeline part of the structure to be made of stainless steel pipes 1.4541 , pipe $6.3 \times 1$;

- the distance (indentation) of the gas distribution pipes from the walls of the vacuum chamber to be $20 \mathrm{~mm}$ - a safe distance from the carousel, ensuring also compactness of the structure in the interior of the chamber. The gas supply pipes to the targets to be located diametrically along a sector line of known length, defined by the position of the end gas supply elements of the system for the 2nd and 4th target;

- the distance (height of placing) of the connecting (the horizontal sectoral) distribution pipe from the bottom of the vacuum chamber to be $-\mathrm{h}=40$, $\mathrm{mm}$;

- the height of the structure from the bottom of the chamber to the top of the gas distribution pipe to measure up to the height of the target frames - 920, mm;

- the openings for supplying the gas through the replaceable gas supply pipes - ø1, mm;

- reliable fixing of the structure during its building-up into the vacuum installation to be provided;

- all overall dimensions to comply with IT14. 
The division of the gas line into sections allows for the inclusion or exclusion of a particular section, depending on the configuration of the targets and the used materials.

2.2. Design and construction of a gas distribution system for modification of the unbalanced magnetron sputtering installation (UDP - 850/4)

In accordance with the set tasks and the technical brief, a gas distribution system is designed and constructed, shown in Fig. 4, and aiming chiefly to regulate the gas supply inside the working space of the installation in specific places and specific quantities.

The positions of the end gas supply pipes (5) through which the gas enters the working space, are determined with the help of the main sections of the gas distribution system. They are welded to each other and form a monolithic structure (Fig. 4a). The supporting elements heels (6) - are rigidly fixed to the monolithic structure, and with the help of them the structure is positioned (fixed) in the chamber (Fig. 4b). The construction of the heels complies with the requirements concerning the vacuum technologies, as well as with their function.

Through the inlet opening (13) the working gases enter the vertical pipe from section 1 (purple). The points of the positions $\mathrm{A}$ and $\mathrm{B}$, from where the gas flow enters the two horizontal sections 2 (blue) and 3 (blue), are determined so that the lengths of the lines along which the gases pass through the pipes of the system until they reach the outlet (end) cross-sections of the three vertical parts of the sections ( 2 and 3 - blue), are equal. The idea in this case is to achieve a certain uniformity of the nature of movement of the gas flow by equating to some extent the resistance that the gas overcomes by passing along the length of the gas distribution system.

Section (3) is constructed as a part of an arc. It is located horizontally to the bottom of the chamber and goes under the heater. This position is determined by attaching it to the bearing heels in especially designed for the purpose grooves. The vertical pipes (4), serving as an extension of the pipeline, are welded at the end of the section and are respectively located at the $2^{\text {nd }}$ and $3^{\text {rd }}$ target.

Section (2) has a kidney-shaped form (bypasses the nests of the thermocouples). The gases, passing through it, are redirected through position $B$.

It is also horizontally placed, along section (3) and below its level. It is welded to the bearing heels through especially made openings (channels) and occupies a minimum distance ( $\sim 3 \mathrm{~mm})$ from the bottom of the vacuum chamber. A vertical pipe (4) is welded at the end of the section, serving as a continuation of the pipeline from the gas distribution system and located at the $4^{\text {th }}$ target.

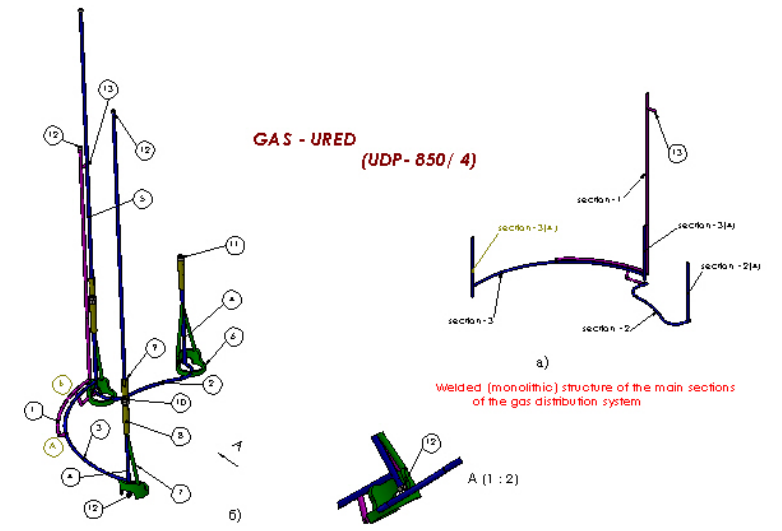

Fig. 4. Gas distribution system for modification of the installation for application of vacuum coatings - (UDP - 850/4):

1 - supply system - part of the gas distribution system

2 - section 2 - provides gas access to target 4

3 - section 3 - provides gas access to targets 2 and 3

4 - vertical tubes (branches) for fixing the gas supply pipes

5 - gas supply pipes at the respective targets

6 - heels (bearing supporting elements of the structure)

7 - braids - reinforcing elements of the structure

8 - lower connection sleeve

9 - upper connection sleeve

10 - fixing nut and counter-nuts Hexagon Hut ISO 4033 -M8 -W - N

11 - stopper - bolt - ISO4762 M8 x 12...12N

12 - stopper - bolt - DIN 912 M5 x 8...8N

13 - tube (branch) for connection to the gas system of the installation (UDP - 850/4)

The three vertical branches (positioning pipes 4) of the monolithic structure are located diametrically (at equal distances) inside the chamber and are shifted at a certain angle to the gas supply (central) pipe at the $3^{\text {rd }}$ target. The gas distribution replaceable pipes (5) are attached to them by means of especially designed sleeves (8 and 9) and with the help of a threaded connection M8. Depending both on the number and on the location of the drilled holes of $ø 1 \mathrm{~mm}$ on them, the amount of gas supplied to the chamber at the respective position is determined. In order to avoid "poisoning" of the targets, it is necessary to orient the position of the gas distribution pipes with the help of the fixing nuts (10) in order that the openings point diametrically toward the interior of the vacuum chamber.

With the help of this gas distribution system it is possible to supply gas separately and independently to each of the selected positions when applying vacuum coatings under the conditions of magnetron (unbalanced) sputtering. If necessary, by means of a stopper (11), the access of gas through a certain position can be blocked, as shown for the position at the $4^{\text {th }}$ target.

The structure is designed to be built-in (fixed) and operate outside the scope of operation of both the carousel and of all moving elements in the structure of the installation (UDP - 850/4) in the vacuum chamber.

\section{CONCLUSIONS}

1. A new design solution for regulating the distribution of the working gases in the vacuum chamber of the 
installation UDP-850/4 for unbalanced magnetron sputtering has been proposed. It will improve the mode and quality of application of multilayer nanocoatings.

2. Constructive documentation for producing the described gas distribution system and instructions for its assembly and installation to the vacuum chamber have been prepared.

3. Based on the technological parameters of the vacuum chamber operating mode and the results of its tests, it is possible to optimize the technological process of applying thin coatings (nanocoatings). This will speed up and optimize the research processes, related to their operational characteristic.

\section{REFERENCES}

[1] Kanev, V., G. Grigorov, High Vacuum, ed. "Technology" 1965.

[2] Mirchev, S., "Fundamentals of vacuum technology", PU "Paisii Hilendarski" - Plovdiv 1986.

[3] White AV, Karpenko GD, Myshkin NK Structure and methods of formation of wear-resistant surface layers, Moscow, Mechanical Engineering, 1991 (In Russian).
[4] Nikitin MM, Vacuum spraying technology and equipment, M, Metallurgiya, 1992 (In Russian).

[5] Danilin BS, Syrchin VK, Magnetron sputtering systems, M, Radio and communication, 1982 (In Russian).

[6] Kuzmichev AI, Magnetron sputtering systems", Kiev, Avers, 2008 (In Russian).

[7] Frolova ES, VG Minaycheva, Vacuum equipment "Handbook", Moscow , Mechanical Engineering, 1985 (In Russian).

[8] Mischev, G., Einfluss der Reibung auf das dynamische Verhalten der geradlinig bewegten Maschinensysteme. $13^{\text {th }}$ International Colloquium Tribology, January 15-17, 2002 Stuttgart/Ostfildern, Germany, ISBN 3-924813-48-5, vol. II, s. 867-873.

[9] Mischev. G., Reibung in geradlinig bewegten Maschinensystemen (Führungen von Werkzeugmaschinen). Fast Print Books, 2020, ISBN:978-619-236-163-1.

[10] Rupetsov, V. , S. Dishliev, G. Mishev, F. Franek, M. Premauer, L. Kolakleva, Research of tribological parameters of multilayer coating Ti/TiN/CrN-ml deposited on 1.2343 steel. BCC, Vol.52, Issue 3, 2020. DOI: 10.34049/bcc.52.3.5236. 